\title{
Comparing Fiducial-Based and Intraoperative Computed Tomography-Based Registration for Frameless Stereotactic Brain Biopsy
}

\author{
Benjamin Saß ${ }^{a}$ Mirza Pojskic ${ }^{a}$ Miriam Bopp ${ }^{a, b}$ Christopher Nimsky a,b \\ Barbara Carl ${ }^{\mathrm{a}, \mathrm{c}}$ \\ ${ }^{a}$ Department of Neurosurgery, University Marburg, Marburg, Germany; ${ }^{\mathrm{b}}$ Marburg Center for Mind, Brain and \\ Behavior (MCMBB), Marburg, Germany; ${ }^{C}$ Helios Dr. Horst Schmidt Kliniken, Wiesbaden, Germany
}

\section{Keywords}

Brain biopsy · Fiducial-based registration - Frameless stereotactic biopsy - iCT-based registration - Low-dose intraoperative computed tomography

\begin{abstract}
Objective: The aim of this work was to compare fiducialbased and intraoperative computed tomography (iCT)based registration for frameless stereotactic brain biopsy. Methods: Of 50 frameless stereotactic biopsies with the VarioGuide, 30 cases were registered as $\mathrm{iCT}$ based and 20 as fiducial based. Statistical analysis of the target registration error (TRE), dose length product, effective radiation dose (ED), operation time, and diagnostic yield was performed. $\boldsymbol{R e}$ sults: The mean TRE was significantly lower using iCT-based registration (mean \pm SD: $0.70 \pm 0.32$ vs. $2.43 \pm 0.73 \mathrm{~mm}, p<$ 0.0001). The ED was significantly lower when using iCTbased registration compared to standard navigational CT (mean \pm SD: $0.10 \pm 0.13$ vs. $2.23 \pm 0.34 \mathrm{mSv}, p<0.0001$ ). Postbiopsy iCT was associated with a significant lower $(p<$ 0.0001 ) ED compared to standard CT (mean \pm SD: $1.04 \pm 0.18$ vs. $1.65 \pm 0.26 \mathrm{mSv}$ ). The mean surgical time was shorter using $\mathrm{iCT}$-based registration, although the mean total operat-
\end{abstract}

karger@karger.com www.karger.com/sfn

Karger $\stackrel{\text { ' }}{5}$

GOPEN ACCESS
(C) 2020 The Author(s)

Published by S. Karger AG, Basel

This is an Open Access article licensed under the Creative Commons Attribution-NonCommercial-4.0 International License (CC BY-NC) (http://www.karger.com/Services/OpenAccessLicense), applicable to the online version of the article only. Usage and distribution for commercial purposes requires written permission. ing room (OR) time did not differ significantly. The diagnostic yield was $96.7 \%$ (iCT group) versus 95\% (fiducial group). Post-biopsy imaging revealed severe bleeding in 3.3\% (iCT group) versus 5\% (fiducial group). Conclusion: iCT-based registration for frameless stereotactic biopsies increases the accuracy significantly without negative effects on the surgical time or the overall time in the OR. Appropriate scan protocols in $\mathrm{iCT}$ registration contribute to a significant reduction of the radiation exposure. The high accuracy of the iCT makes it the more favorable registration strategy when taking biopsies of small tumors or lesions near eloquent brain areas.

(c) 2020 The Author(s)

Published by S. Karger AG, Basel

\section{Introduction}

In the second half of the 20th century stereotactic biopsies evolved to a safe standard procedure in the diagnosis of brain tumors [1] and became an integral part of many other neurosurgical procedures [2-9]. Subsequently developed image-guided frameless stereotactic systems proved to reach targeting errors and clinical results similar to those of frame-based stereotaxy $[10,11]$. Previous reports on the frameless VarioGuide system 
Axial $A$ Nasigaston 2.4 .4 .5
$29.082018,1254$

.

R

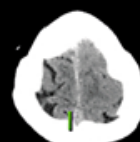

$-\infty$

p

Carefully check the accuracy by touching landmarks with the pointer. Do you accept the registration accuracy?

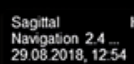

Narogation 2.4

L R
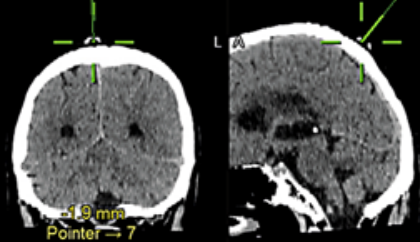

a

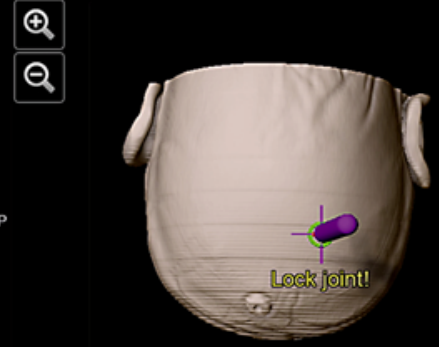

(9)
Step 3: Adjust Joint 1
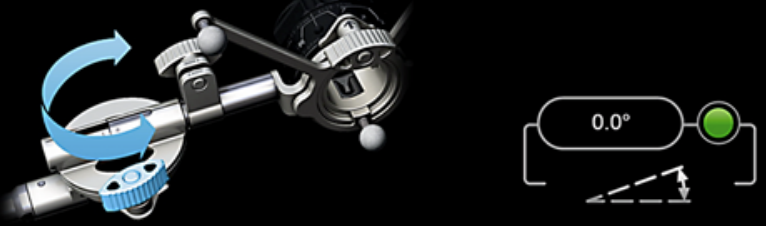

Lock joint!

1. Loosen the highlighted joint and move it back and forth until the number appears.

Step 5: Adjust Joint 3
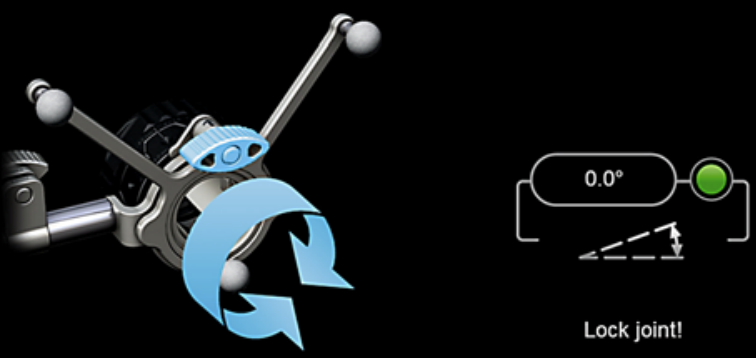

Lock joint!
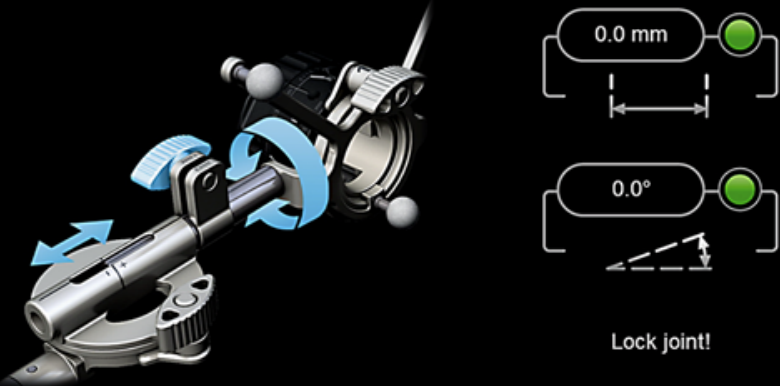

b Align the VarioGuide roughly with the trajectory and move it close to the head.

Step 4: Adjust Joint 2

d

Step 6: Review Alignment

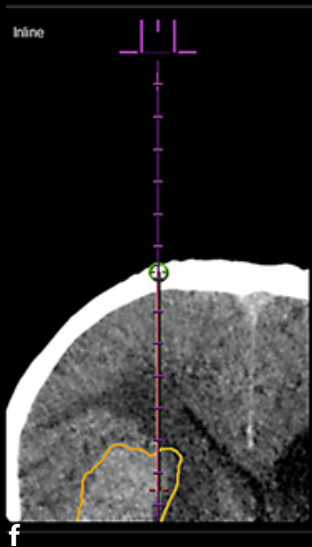

Joint 1

$0.0^{\circ}$

Joint 2

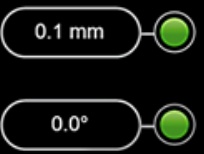

Joint 3

$0.0^{\circ}$

Fig. 1. The accuracy is checked by touching landmarks with the pointer, then the registration is verified (a). After coarse positioning of the head of the unit (b) the three joints (c-e) are adjusted close to $0^{\circ}$. A review of the alignment is displayed, pointing out the calculated entry and target deviation (f).

(Brainlab AG, Munich, Germany) focused on accuracy, diagnostic yield, and complications after standard registration (Z-touch, anatomical landmarks, fiducials) [1214]. In frameless stereotaxy registration based on ana- tomical landmarks or skin fiducials provides comparable target registration errors and therefore may be used interchangeably depending on the surgeon's preference [15]. Nonetheless, standard registration procedures are 
highly user dependent. The introduction of modern intraoperative computed tomography (iCT), such as the O-arm (Medtronic) [16] or the AIRO-CT (Brainlab), allows user-independent automatic registration with high accuracy and low radiation exposure in cranial procedures, as we reported earlier [17]. Recently, iCT-based registration for stereotactic surgery has been described as a safe, reliable, and accurate alternative to standard stereotactic procedures [18]. However, little is known about the implementation of iCT into frameless stereotactic biopsy procedures. To our knowledge, there is a single report on a VarioGuide supported drainage of a brainstem abscess using the AIRO-CT scanner for registration [19], but larger series of this approach are lacking and there are no data available concerning registration accuracy, radiation exposure, operation time, and diagnostic yield of iCT-based frameless biopsies. With the intention of closing this lack of data, we present our experience of 50 cases of frameless stereotactic biopsies with the VarioGuide system comparing fiducial with iCT-based registration. We hypothesized that the implementation of iCT-based registration for frameless stereotactic biopsies is associated with a higher registration accuracy, a lower radiation exposure, a comparable diagnostic yield, and does not prolong the whole operative procedure.

\section{Materials and Methods}

In this retrospective analysis all cases of frameless stereotactic biopsies with the VarioGuide system obtained between May 2017 and April 2019 were included, giving a total of 50 patients (25 females and 25 males; age range from 7 to 93 years). Fiducial-based registration was used in 20 and iCT-based registration in 30 cases. In all cases preoperative imaging revealed a brain lesion of unknown etiology, and an interdisciplinary tumor board recommended biopsy. The decision on the registration method was only based on the availability of iCT and trained staff, patient-related criteria were not taken into account. No case was excluded. Informed written consent was obtained from all patients or their appropriate family members. We obtained ethical approval for prospective archiving of all relevant clinical and technical data with no need for further approval of retrospective analysis.

\section{Preoperative Imaging and Registration}

All patients underwent magnetic resonance imaging (MRI) for diagnostic purposes prior to surgery. In the fiducial-based registration group at least 7 skin fiducials were placed on the patient's head and a cranial fine-cut 3D-CT scan was performed in the Department of Neuroradiology on the day before surgery, except in case No. 1 and 7, where a navigation-MRI was implemented in the work-flow. Preoperative CT and MRI images were rigidly registered applying the image fusion software element (Brainlab). After

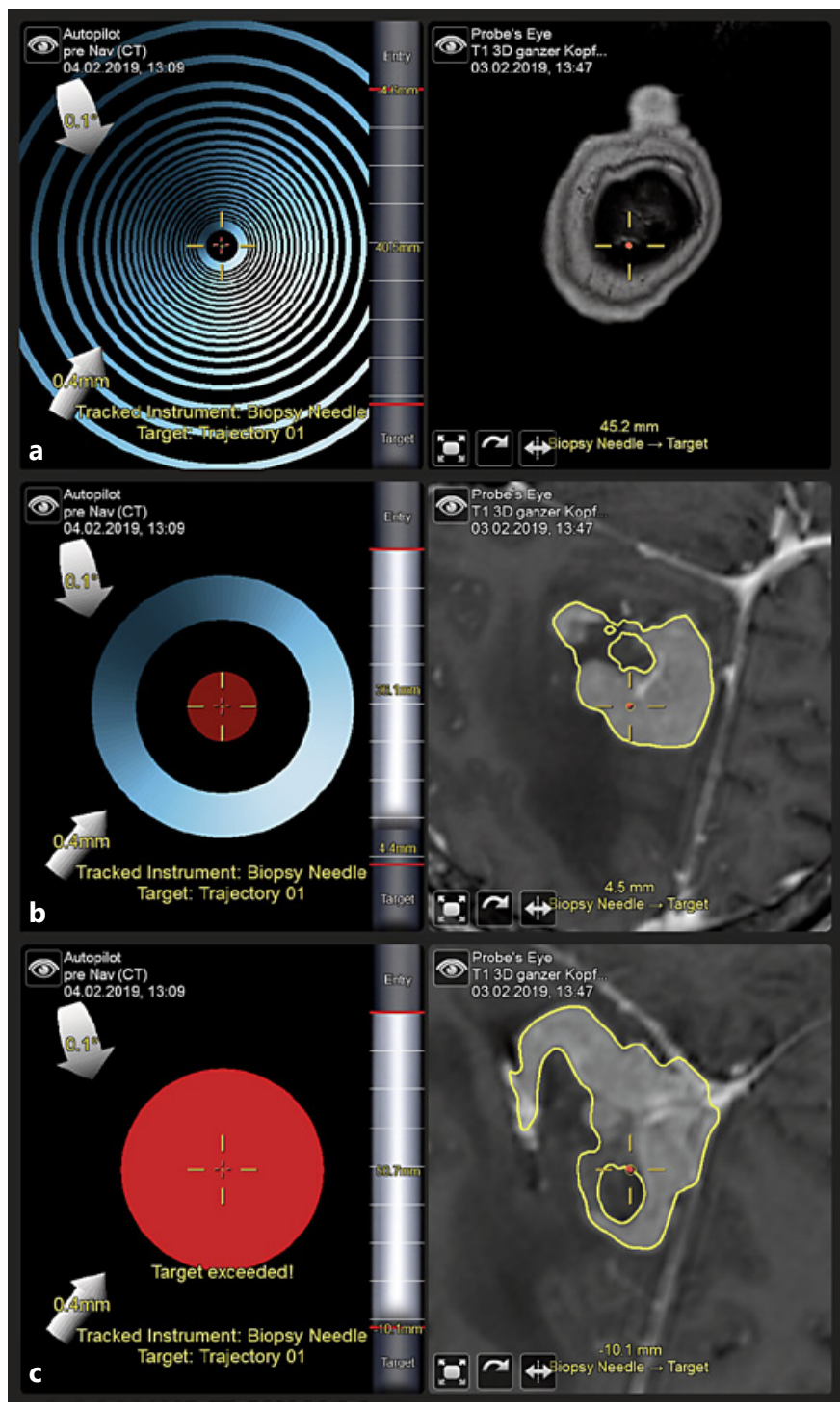

Fig. 2. A precalibrated, navigated biopsy needle is inserted. The navigation monitors display the position of its entry (a), whilst advancing (b), and when reaching and exceeding the target position (c). The autopilot (left in $\mathbf{a}-\mathbf{c}$ ) gives a straightforward feedback on the penetration depth of the biopsy needle, the probe's eye view (right in $\mathbf{a}-\mathbf{c}$ ) demonstrates the needle within the planned trajectory.

placing the head in a standard 3-pin head holder, the reference array was attached to the head clamp and the navigation camera aligned in a way that allowed the detection of the reference array and navigation pointer when placed on the head. Using the registration module of the navigation software, patient registration was then established by placing the tip of the navigation pointer in the divot of each fiducial, except one used to determine the target registration error (see below).

In the iCT-based registration group the patient's head was attached to the operating room (OR) table with a radiolucent 

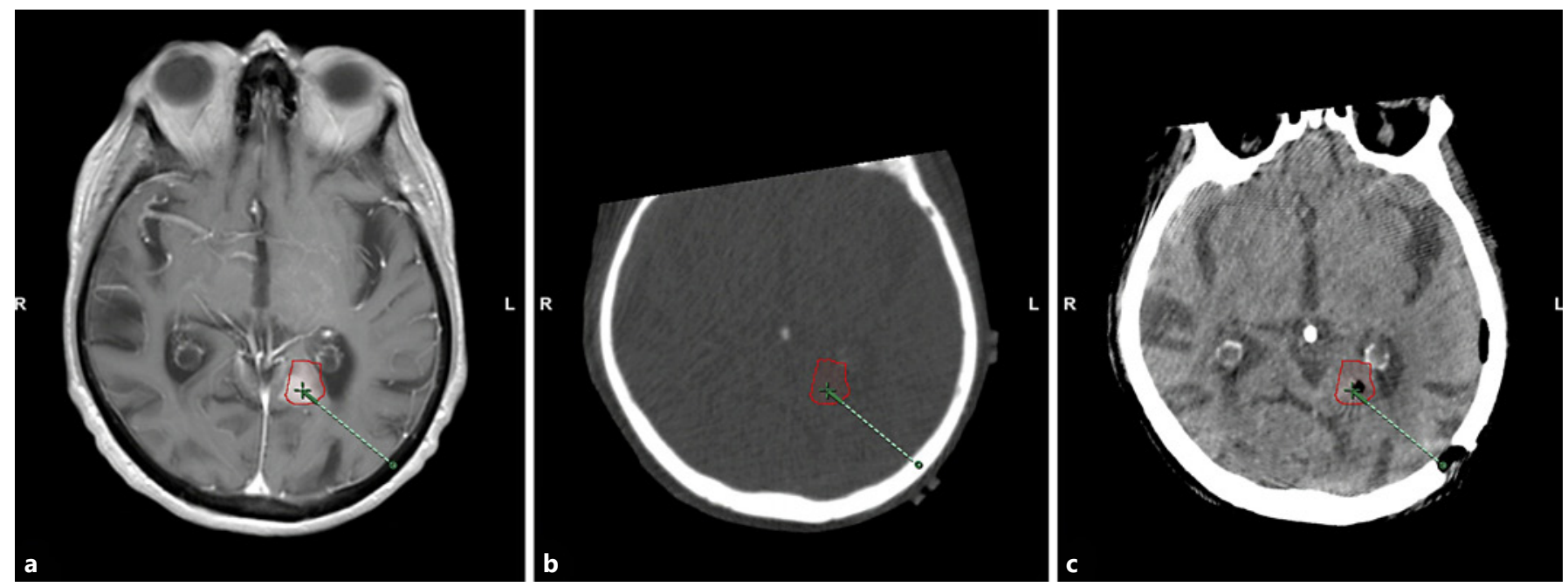

Fig. 3. Documentation of the correct biopsy site: fusion of the preoperative MRI (a), registrational CT (b), and postoperative CT (c) show the instilled air within in the target volume.

skull clamp and standard metallic pins, which were placed outside the scanning area. Although not needed for registration, three skin fiducials were placed within the planned scan section allowing accuracy measurement later. A radiolucent reference array was fastened to the head holder, the OR table was rotated by $90^{\circ}$, and an $\mathrm{iCT}$ registration scan carried out $(60-\mathrm{mm}$ scan length). For iCT scanning a 32-slice movable CT scanner (AIRO, Brainlab) was used, closely integrated in the navigation setup consisting of a ceiling-mounted double monitor navigation system in combination with a wall-mounted dual display system for consecutive visualization of co-registered images and operation of the stereotactic Brainlab elements software. It is mandatory that during the scanning process the reference array and reflective markers on the scanner are in the field of view of the navigation camera. A lateral scout and different low-dose scan protocols were applied; details of the setup have previously been published [17]. After automatic registration, the iCT registration scan and preoperative MRI datasets were rigidly registered as in the fiducial group.

In order to determine the registration accuracy, the tip of the navigation pointer was placed in the divot of a fiducial marker, which was not part of the registration process, and the target registration error (TRE) was calculated as the Euclidian offset of the pointer tip.

\section{Procedure}

In all cases, biopsy targets were identified in preoperative imaging and marked with the smart brush tool element (Brainlab), which allowed the measurement of the total target volume. Optimal trajectories were planned preoperatively by determining the entry and target point with the trajectory tool element (Brainlab), which could be adjusted or re-planned in the OR, if needed.

All procedures were performed under general anesthesia, with the patients placed in a prone or supine position. After the registration procedure, the preplanned entry point was identified with the navigation pointer and marked on the skin. After surgical skin preparation and draping the VarioGuide arm was attached to the head clamp. Following skin incision, burr hole trepanation, and dura opening, the navigated head of the VarioGuide was roughly placed over the entry point. The software setup assistant was opened and provided guidance through the fine adjustment by exact positioning of the three lockable joints in the head of the unit (Fig. 1). A navigated, precalibrated $1.8-\mathrm{mm}$ disposable side cutting biopsy needle (Pajunk, Geisingen, Germany) was inserted through the head of the VarioGuide and the advancement of the needle was displayed in real-time on the navigation monitors (Fig. 2). Once the needle reached the target point, sequential biopsies were taken, and smear preparations of the sample tissue were performed right away in the OR by the neuropathologist with instant feedback to the neurosurgeon. The biopsy needle was only repositioned, and a second series of biopsies taken, when the neuropathologist could not detect pathological tissue in the first probe.

Finally, as a control in the iCT group, $0.5 \mathrm{~mL}$ of air were insufflated into the site of biopsy (Fig. 3). After wound closure, either a second iCT scan (iCT group) was performed as a full-dose CT with a restriction of the scan length to $60 \mathrm{~mm}$ (except case No. 9), which allowed an overall reduction of the radiation exposure, or the patient received a conventional cranial CT scan in the Department of Neuroradiology (fiducial group).

The dose length product (DLP) refers to a phantom with a diameter of $16 \mathrm{~cm}$. For an estimation of the effective radiation dose (ED), current ED/DLP conversion factors were estimated to be 2.4 $\mu \mathrm{Sv} / \mathrm{mGy} \cdot \mathrm{cm}$ for head scans $[20]$.

\section{Statistical Analysis}

Statistical analysis was performed with GraphPad Prism 8.1.2 for MacOS. Parametric data were analyzed with the $t$ test with Welch's correction under the assumption of unequal standard deviation (SD), for non-parametric data the Mann-Whitney test was used. Fisher's exact test was used in the analysis of contingency tables. A $p$ value $<0.05$ was considered statistically significant. 


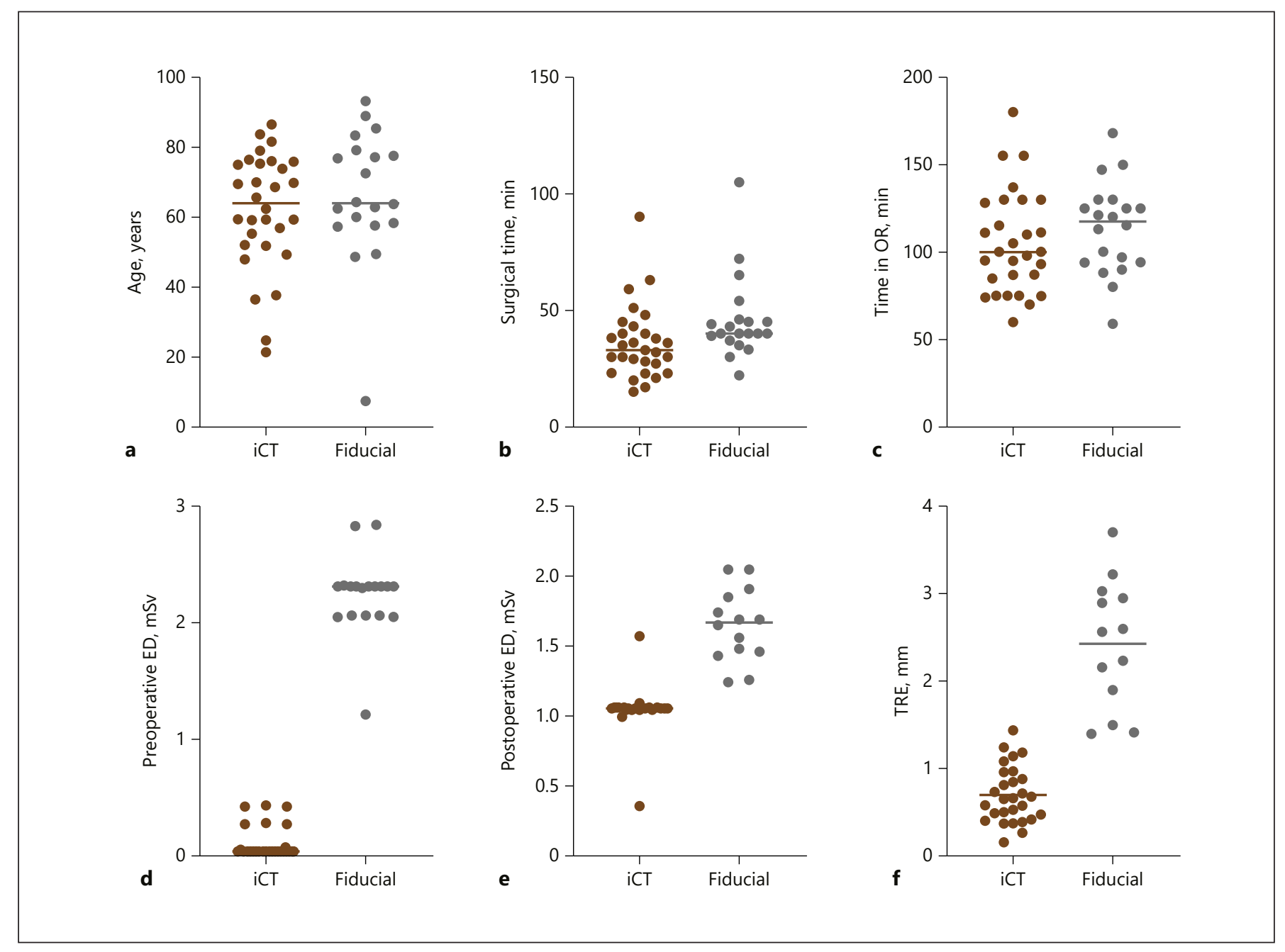

Fig. 4. Scatter dot plots with lines at the median (a-e) or at the mean (f). a The mean patient age in the iCT group was 62.0 years $(n=30)$ versus 66.3 years in the fiducial group $(n=20)$, the median in both groups was 64.0 years. $\mathbf{b}$ The median surgical time in the iCT group was $33 \mathrm{~min}(n=29)$ versus $40 \mathrm{~min}$ in the fiducial group $(n=20)$. This was statistically significant $(p=0.0094$, twotailed Mann-Whitney test). c The median time in the OR in the iCT group was $100 \mathrm{~min}(n=29)$ versus $117.5 \mathrm{~min}$ in the fiducial group $(n=20)$. This was not statistically significant $(p=0.23$, twotailed Mann-Whitney test). $\mathbf{d}$ The preoperative radiation exposure was significantly lower using iCT for registration $(p<0.0001$, two-

\section{Results}

The mean patient age in the iCT group was 62.0 years (range 21.4-86.6), 15 patients were females and 15 were males (Fig. 4a). Histological work-up revealed 16 glioblastomas, 1 diffuse midline glioma, 1 anaplastic astrocytoma, 1 diffuse astrocytoma, 5 diffuse large B-cell lym- tailed Mann-Whitney test): iCT group $(n=28)$, median DLP $=17$ $\mathrm{mGy} \cdot \mathrm{cm}, \mathrm{ED}=0.04 \mathrm{mSv}$ versus fiducial group $(n=18)$, median $\mathrm{DLP}=961 \mathrm{mGy} \cdot \mathrm{cm}, \mathrm{ED}=2.31 \mathrm{mSv}$. e The postoperative radiation exposure was significantly lower with iCT registration (two-tailed Mann-Whitney test): iCT group $(\mathrm{n}=26)$, median DLP $=437$ $\mathrm{mGy} \cdot \mathrm{cm}, \mathrm{ED}=1.05 \mathrm{mSv}$ versus fiducial group $(n=14)$, median $\mathrm{DLP}=695 \mathrm{mGy} \cdot \mathrm{cm}, \mathrm{ED}=1.67 \mathrm{mSv}$. f Regarding accuracy, the TRE was significantly lower using iCT for registration $(p<0.0001$, two-tailed unpaired t test with Welch's correction); iCT group $(n=28): 0.70 \pm 0.32 \mathrm{~mm}$ (mean $\pm \mathrm{SD})$ versus fiducial group $(n=$ 13): $2.43 \pm 0.73 \mathrm{~mm}$. of progressive multifocal leukoencephalopathy, 1 cerebral cavernous hemangioma, 1 case of axonal degeneration after injury, and 1 inconclusive diagnosis. This amounts to a diagnostic yield of $96.7 \%$, which is slightly higher but not statistically significant than in the fiducial-based registration group (95.0\%), encompassing 10 


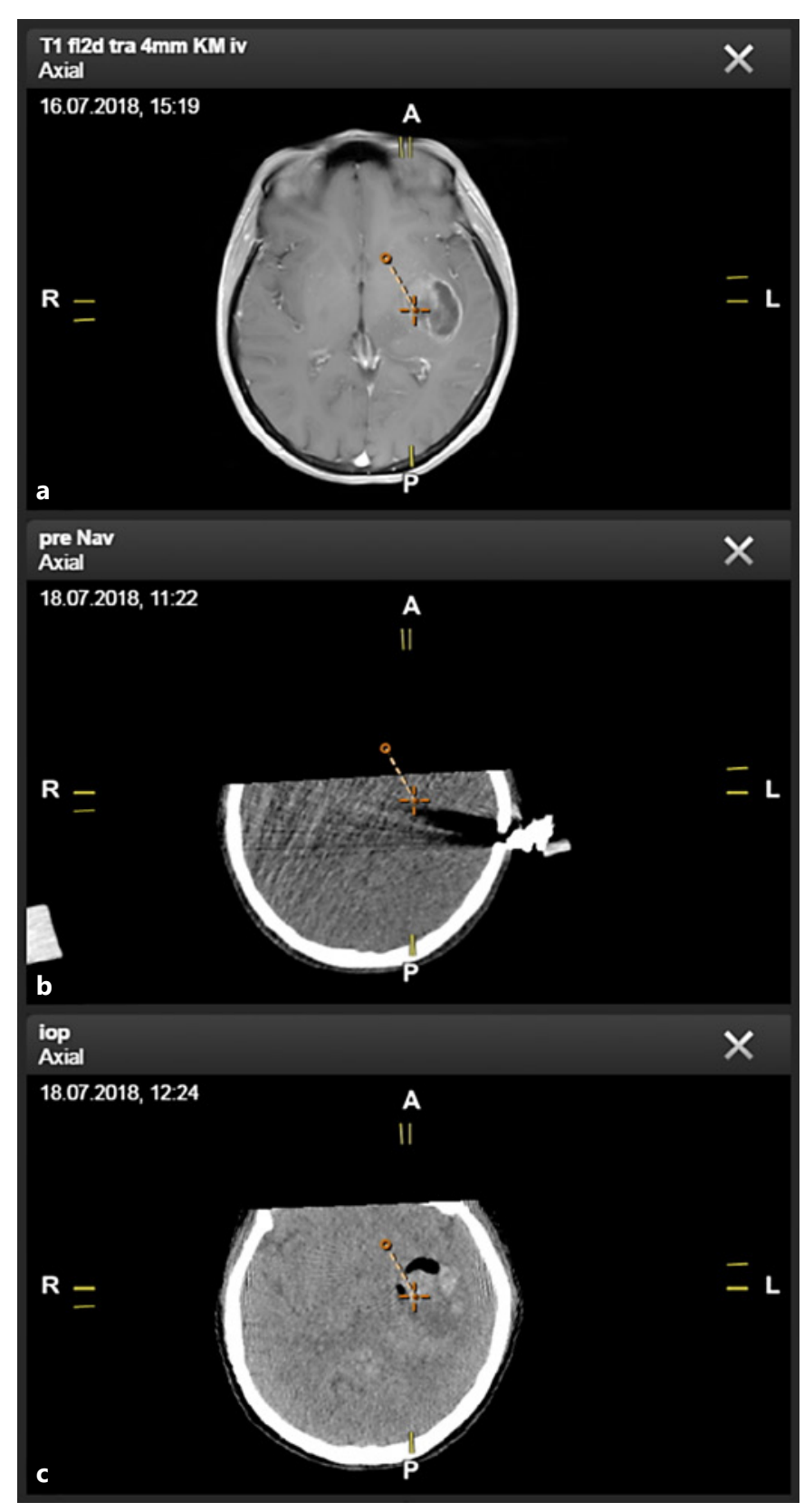

Fig. 5. Case 17: preoperative MRI (a) and navigational iCT (b) do not show any bleeding. The corresponding axial slices of the postoperative iCT (c) document a hemorrhage at the biopsy site.

females and 10 males at a mean age of 66.3 years (range 7.4-93.2). Here, in 7 cases a glioblastoma and in 1 case an anaplastic astrocytoma were diagnosed, and 6 patients suffered from diffuse large B-cell lymphoma. Furthermore, histological examination showed 1 brain abscess, 1 case of echinococcosis, 1 case of progressive multifocal leukoencephalopathy, 1 metastasis of small cell lung cancer, 1 case of resorptive tissue after intracerebral hemorrhage, and 1 suspected neuronal heterotopia (the final diagnosis remained unclear). The mean target volume encompassing the whole lesion was 33.2 $\mathrm{cm}^{3}$ in the iCT group and $16.6 \mathrm{~cm}^{3}$ in the standard registration group.

The mean duration of the operation was 36 min (median 33) in the iCT group (case No. 47 was excluded, since the concept was changed to open surgery intraoperatively) compared to $46 \mathrm{~min}$ (median 40 ) with standard registration. Thus, the surgery was performed significantly faster using iCT-based registration ( $p=0.0094$, two-tailed Mann-Whitney test; Fig. 4b). Although the mean total time in the OR, which includes positioning of the patient and the registration procedure, was shorter in the iCT group (mean 105 vs. 114 min, median 100 vs. 117.5 min), the difference was not significant ( $p=0.2300$, two-tailed Mann-Whitney test; Fig. 4c).

Analysis of the preoperative radiation exposure (Fig. 4d), comparing the navigational CT to the intraoperative iCT scan, showed a significant lower exposure in the iCT group $(p<0.0001$, two-tailed Mann-Whitney test). Whilst with the navigational CT a median DLP of $961 \mathrm{mGy} \cdot \mathrm{cm}$ (mean $\pm \mathrm{SD} 931 \pm 142$ ) equivalent to a median $\mathrm{ED}$ of $2.31 \mathrm{mSv}$ (mean \pm SD $2.23 \pm 0.34$ ) was applied, the median DLP was only $17 \mathrm{mGy} \cdot \mathrm{cm}$ (mean \pm SD $45 \pm$ 55 ) and median ED $0.04 \mathrm{mSv}$ (mean \pm SD $0.10 \pm 0.13$ ) in the iCT group (in case No. 1 and 7 a navigational MRI was performed, in case No. 13 and 31 no data on the DLP were available, thus they were excluded). The scan length was $60 \mathrm{~mm}$ in the iCT group, but ranged from 140 to $209 \mathrm{~mm}$ (mean $168 \mathrm{~mm}$ ) in the fiducial group.

Additionally, in the postoperative control DLP and ED were lower using iCT (median DLP $437 \mathrm{mGy} \cdot \mathrm{cm}$; mean $\pm \mathrm{SD} 434 \pm 73 \mathrm{mGy} \cdot \mathrm{cm}$; median ED $1.05 \mathrm{mSv}$; mean \pm SD $1.04 \pm 0.18 \mathrm{mSv}$ ) compared to a control CT in the Department of Neuroradiology (median DLP $695 \mathrm{mGy}{ }^{\circ} \mathrm{cm}$; mean \pm SD $687 \pm 108 \mathrm{mGy} \cdot \mathrm{cm}$; median ED $1.67 \mathrm{mSv}$; median \pm SD $1.65 \pm 0.26 \mathrm{mSv}$ ), which was also statistically highly significant $(p<0.0001$, two-tailed MannWhitney test; Fig. 4e). Again, the iCT scan was restricted to a length of $60 \mathrm{~mm}$ (except case No. 9: $90 \mathrm{~mm}$ ), whilst the scan length in the fiducial group ranged from 126 to $173 \mathrm{~mm}$ (mean 154). No postoperative CT was performed in cases $1,2,3,7,25,35$, and 37; therefore, these were excluded from the analysis. Cases 13,31 , and 47 were excluded because they were registered with iCT but received a conventional post-biopsy CT scan.

The second iCT scan revealed an intracranial hemorrhage at the biopsy site in case No. 17 (Fig. 5), which led
Saß/Pojskic/Bopp/Nimsky/Carl 

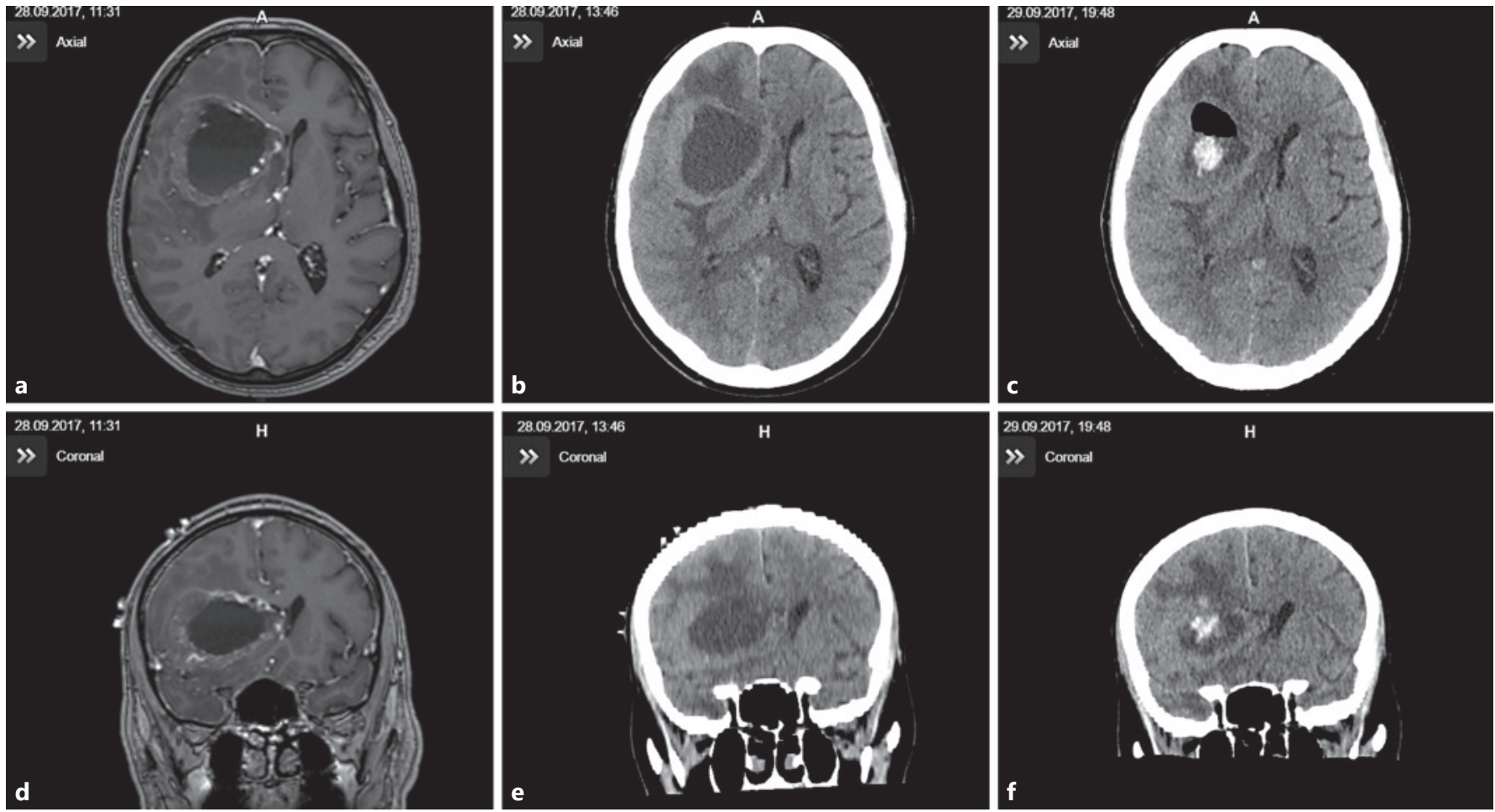

Fig. 6. Illustrative case (No. 6) of a severe hemorrhage detected in postoperative CT in the fiducial group: axial slices of the preoperative MRI (a), CT (b), and the corresponding slices in the postoperative CT (c). d-f Coronal slices of preoperative MRI and CT, and postoperative CT.

to a mild hemiparesis, but did not demand further treatment. Case No. 41 developed a transient aphasia, although no postoperative bleeding was found. In case No. 13 a thin layer of blood and in case No. 31 a minimal hemorrhage at the biopsy site were detected, both received a conventional CT control. Case No. 13 was disoriented post-biopsy, while case No. 31 did not develop new deficits. In cases $8,9,10,16,17,18,20,21,23,25,26,32,40,41,43,45$, and 46 further imaging was performed within a few days for different reasons (e.g., irradiation planning), none of them revealing new bleeding at the biopsy site.

In the fiducial-based registration group, minor postoperative hemorrhages were detected in cases 5 (intraventricular), 24, 34, and 44, and a severe bleeding in case no. 6 (Fig. 6). None of them were clinically apparent.

In all cases that received postoperative imaging, the insufflated $0.5 \mathrm{~mL}$ of air at the biopsy site was able to document the sample being taken within the planned target area. Interestingly, in case No. 9 a major hemorrhage within the tumor was identified in the preoperative iCT scan, which was not documented in the earlier imaging, and led to a rapid deterioration of the patient.

iCT-Based Frameless Stereotactic Brain Biopsy
There was a highly significant difference $(p<0.0001$, two-tailed unpaired $t$ test with Welch's correction) of the target registration error using $\mathrm{iCT}$ for patient registration (Fig. 4f), which was $0.70 \pm 0.32 \mathrm{~mm}$ (mean $\pm \mathrm{SD}$ ), compared to the accuracy in the fiducial-based registration group (mean \pm SD $2.43 \pm 0.73 \mathrm{~mm}$ ); however, 9 cases had to be excluded from the analysis due to a lack of an adequate intraoperative documentation to allow reliable retrospective analysis (cases $7,14,17,27,29,31,34,36$, and 44).

\section{Discussion}

In our retrospective analysis of 50 cases, we found the application of iCT-based automatic, user-independent registration to be associated with a registration error as low as $0.70 \pm 0.32 \mathrm{~mm}$, whilst it was $2.43 \pm 0.73 \mathrm{~mm}$ in the fiducial-based registration group. Additionally, the radiation exposure using iCT for registration was reduced to a DLP of $17 \mathrm{mGy} \cdot \mathrm{cm}$, which is equivalent to an estimated $\mathrm{ED}$ of $0.04 \mathrm{mSv}$, and thus far lower when compared to a 
standard navigational fiducial CT in the Department of Neuroradiology (median DLP $961 \mathrm{mGy} \cdot \mathrm{cm}$; ED 2.31 $\mathrm{mSv})$. The implementation of $\mathrm{iCT}$ into the registration procedure turned out to be uncomplicated and user friendly, resulting in a significantly shorter mean surgical time (36 vs. $46 \mathrm{~min}$ ), whereas the mean total time in the OR was only slightly shorter (105 vs. $114 \mathrm{~min}$, not significant). Regardless of the mode of registration the diagnostic yield was exceptional high (iCT group $96.7 \%$, fiducial group 95\%), and severe hemorrhage was found in only 1 case in each group.

Studies on the accuracy of navigated guided biopsies reach as far back as 1999, when Dorward et al. [21] reported a mean localization error in phantom studies of $1.3 \pm 0.6 \mathrm{~mm}$, but revealed a noticeably greater mean Euclidean error of $4.8 \pm 2 \mathrm{~mm}$ as measured in postoperative MRI. In 2009 the newly developed VarioGuide biopsy system was assessed by Ringel et al. [12], who performed phantom-based accuracy measurements and calculated a mean localization error of $1.44 \pm 0.98 \mathrm{~mm}$. They found a maximum deviation of $5 \mathrm{~mm}$ in one direction and therefore assumed a spherical lesion of a diameter of $10 \mathrm{~mm}$ (and a total volume of $0.524 \mathrm{~cm}^{3}$ ) to be biopsied with a $100 \%$ probability that the needle actually entered the target volume. Bradac et al. [14] studied the accuracy of the VarioGuide system compared to frame-based stereotaxy in the clinical setting by obtaining intraoperative MRI scans after wound closure, and found a mean distance between the planned and real target of $2.90 \pm 1.26 \mathrm{~mm}$ in the VarioGuide group, which did not significantly differ from the mean offset of $2.65 \pm 1.12 \mathrm{~mm}$ in frame-based stereotaxy. In our analysis, we found a similar accuracy using fiducial-based registration $(2.43 \pm 0.73 \mathrm{~mm})$, but a far lower target registration error $(0.70 \pm 0.32 \mathrm{~mm})$ with iCT-based automatic registration, providing strong evidence for its superiority in terms of accuracy. Yet, one has to point out that we used a different method for calculating the localization error, as we did not compare the planned and real targets in the postoperative imaging. Although we instilled $0.5 \mathrm{~mL}$ of air at the biopsy site, which allowed us an instant control to determine we actually reached the target volume, we found it to be inconvenient and imprecise as a method of accuracy measurement and, therefore, we calculated the TRE with fiducial markers as mentioned above. This approach has been published in a similar manner before [22-24], but Mascott et al. [25] pointed out that the measurements might be prone to error due to fiducial shifting and the distance between the skin-attached fiducial and the actual target. To avoid inaccuracy due to the aforementioned problem, they placed small drill holes as targets in the cranium and additionally $1-\mathrm{mm}$ titanium hemoclips along the falcine or basal dura for accuracy measurement. However, we found these measures not to be feasible in the setting of frameless stereotactic biopsies because the surgical site is limited to a small skin incision and a burr hole. Additionally, we believe the suspected errors in both groups to be similar, allowing a comparison and conclusion on the accuracy. The shorter mean surgical time in the iCT group (36 vs. $46 \mathrm{~min}$ ), which was overall similar to other reports $(42-49 \mathrm{~min})[26,27]$, can hardly be explained by the mode of registration, because there is no difference in the procedure once the registration is finished. Although the VarioGuide is straightforward to use [12], it can be assumed that the surgical time is likely biased by different surgeons' experiences with the system. This might also explain the non-significant differences in the mean total time in the OR (105 vs. $114 \mathrm{~min}$ ), which in both groups was substantially shorter compared to a report on older frameless stereotactic systems (185 $\mathrm{min}$ ) [28]. However, these data suggest that the implementation of iCT into the registration process does not delay the whole procedure and registration time is probably shorter and more efficient than in the fiducial-based approach.

Performing an iCT scan for registration is associated with radiation exposure to the patient. The applied lowdose CT accounted for only an ED of $0.04 \mathrm{mSv}$, which is similar to the ED of a single posteroanterior X-ray examination of the chest [29], revealing a tremendous difference to a standard navigational fiducial CT with an ED of $2.31 \mathrm{mSv}$ in the Department of Neuroradiology. However, different approaches might reduce radiation in standard fiducial registration procedures. A preoperative navigation-MRI, as implemented in case No. 1 and 7, could avoid any radiation exposure entirely, but due to restricted MRI capacity is reserved for exceptional cases only. There is certainly great potential in lowering the exposure to the patient from navigational fiducial CT by the application of low-dose protocols and adjustment of the scanning range. The latter will be difficult realize because the neuroradiologist is not very familiar with neurosurgical approaches and positioning of the patient, which is a prerequisite for the shortening of the scan length. Additionally, the navigation fiducial CT is typically performed 1 day prior to surgery and is therefore prone to fiducial shifting up to surgery, making it susceptible for registration errors. Taken together, the application of iCT after patient positioning offers the ideal setting for the reduction of radiation exposure and minimizes the risk of registration errors.
Saß/Pojskic/Bopp/Nimsky/Carl 
One approach to avoid radiation exposure at all is the implementation of intraoperative MRI (iMRI) into the biopsy procedure, if available. Different scenarios of how iMRI can be integrated into biopsy procedures have been described. Moriarty et al. [30] reported an excellent target localization in a series of phantom experiments and 68 frameless stereotactic biopsies using iMRI and pointed out the advantage of real-time imaging permitting the confirmation that the biopsy needle actually reached the target, but iMRI has also been described to be safe and effective when integrated in frame-based stereotactic biopsies [31]. The real-time assessment of the needle position when using iMRI allows the evaluation of brain shift during the procedure or detection and compensation of a needle deviation from the planned trajectory [32]. Standard frame-based, frameless stereotactic, or iMRI-guided biopsies proved to have a comparable diagnostic yield [32]. It is noteworthy that the use of iMRI in frameless stereotactic biopsies prolongs the whole procedure on the one hand [33], but on the other hand possibly reduces the length of hospital stay and lowers the risk of serious complications when compared to standard frame-based or frameless stereotactic biopsy techniques [32]. When discussing the application of iMRI-guided biopsies, the overhead costs of MRI acquisition and maintenance should not be underestimated [32], but, nevertheless, iMRI-guidance can be recommended for the minority of those who have already integrated an iMRI into their surgical theater [31].

Interestingly, in case No. 9, preoperative iCT was able to detect a major hemorrhage within the tumor, which was not seen on prior MRI scans, and led to a rapid deterioration of the patient. This would most likely have been diagnosed as an intraoperative complication otherwise. Since this was one of the early cases, when we were still in the process of a stepwise reduction of the dose to a minimum that would still allow a reliable registration performance, the effective dose was rather high $(0.43 \mathrm{mSv})$. Applying the current low-dose protocols would render it highly unlikely that the hemorrhage would have been detected.

Another advantage of $\mathrm{iCT}$ is the possibility to perform a first imaging control in the OR and instantly rule out adverse events or, if applicable, to react promptly. The restriction to a scan length of $60 \mathrm{~mm}$, focused on the target volume, led to a median ED of 1.05 compared to 1.67 $\mathrm{mSv}$ when the control was performed in the Department of Neuroradiology. Yet only two hemorrhages were identified in the postoperative iCT, one of them severe. We found an overall bleeding rate of $16 \%$ in the VarioGuide

iCT-Based Frameless Stereotactic Brain Biopsy procedure, whereby it was $10 \%$ in the iCT group and $25 \%$ in the conventional registration group, but not a single case demanded revision. Notably, in both groups only one hemorrhage (approx. 3.3 and 5\%, respectively) was classified as severe, which is comparable the findings of others $[12,14]$. Only 2 cases in which we found postoperative bleeding developed neurological deficits (disorientation, mild hemiparesis), and 1 patient showed a transient aphasia without correlate in the postoperative imaging.

In recent years, the high diagnostic yield of different navigated frameless biopsy techniques has become evident, ranging from 87 to $100 \%$ [34]. Previous studies on VarioGuide biopsies reported a diagnostic yield ranging from 90 to $94.6 \%[12,26,27,13,35,36]$. Here, we found a slightly higher diagnostic rate of $96.7 \%$ with iCT registration and 95\% with standard registration, probably as a result of the consequent implementation of intraoperative examination of the specimens into the workflow.

We are aware of the limitations of this study, which are mainly due to its retrospective nature, such as the missing randomization and blinding, and differences in quality and quantity of documentation of the procedure and the patient outcome. The lack of data led to an exclusion of cases, for example in the analysis of accuracy in the standard registration group, whilst the documentation was thorough in the iCT group. However, the number of excluded cases did not reach a level that affected the overall reliability of the statistical analysis. Another shortcoming of this study is the fact that different surgeons where involved in the procedures, and the impact of this on the measured parameters remains somewhat unclear. It seems unlikely that there is a significant effect on the registration accuracy, but parameters like the operation time or overall time in the OR are probably influenced by the surgeon's experience. Thus, as stated above, care has to be taken when interpreting these data. Taken together, despite these limitations, we believe that the data acquired are strong enough to allow a reliable conclusion.

\section{Conclusion}

$\mathrm{iCT}$ as a registration device for frameless biopsy is superior to fiducial-based registration in terms of accuracy, whereas the radiation exposure is significantly reduced when compared to a standard registration CT. The registration procedure is straightforward in use, user indepen- 
dent, and has no negative effect on the operating time or overall time in the OR. The diagnostic yield of the VarioGuide biopsies was exceptionally high in both groups, implying both registration procedures to be adequate in most cases. However, its superiority in accuracy suggests the use of iCT registration, when available, especially for the biopsy of small target volumes or when the lesion is located near eloquent areas.

\section{Statement of Ethics}

All procedures performed in studies involving human participants were in accordance with the ethical standards of the institutional and/or national research committee (Ethical Committee of the Medical Faculty of Philipps University, Marburg, Germany) and with the 1964 Helsinki declaration and its later amendments or comparable ethical standards.

\section{Conflict of Interest Statement}

PD Dr. B. Carl and Prof. Dr. med. Christopher Nimsky declare that they have received speaker fees from Brainlab. They have not received any separate financial support for this contribution. All other authors have no conflicts of interest to declare.

\section{Funding Sources}

No funding was involved in this work.

\section{Author Contributions}

C.N. and B.C. conceived and supervised the presented study. B.S and M.B. collected the data, which were statistically evaluated by B.S. The manuscript was written by B.S., who was encouraged and supported by M.P. All authors discussed the results of this study and contributed to the final version of the manuscript.

\section{References}

1 Krieger MD, Chandrasoma PT, Zee CS, Apuzzo ML. Role of stereotactic biopsy in the diagnosis and management of brain tumors. Semin Surg Oncol. 1998 Jan-Feb;14(1):13-25.

2 D'Angelo V, Gorgoglione L, Catapano G. Treatment of symptomatic intracranial arachnoid cysts by stereotactic cyst-ventricular shunting. Stereotact Funct Neurosurg. 1999;72(1):62-9.

3 Niranjan A, Witham T, Kondziolka D, Lunsford LD. The role of stereotactic cyst aspiration for glial and metastatic brain tumors. Can J Neurol Sci. 2000 Aug;27(3):229-35.

4 Cossu M, Cardinale F, Castana L, Citterio A, Francione S, Tassi L, et al. Stereoelectroencephalography in the presurgical evaluation of focal epilepsy: a retrospective analysis of 215 procedures. Neurosurgery. 2005 Oct; 57(4):706-18.

5 Moorthy RK, Rajshekhar V. Management of brain abscess: an overview. Neurosurg Focus. 2008;24(6):E3.

6 Hemm S, Wårdell K. Stereotactic implantation of deep brain stimulation electrodes: a review of technical systems, methods and emerging tools. Med Biol Eng Comput. 2010 Jul;48(7):611-24.

7 Pizzolato G, Mandat T. Deep brain stimulation for movement disorders. Front Integr Nuerosci. 2012 Jan;6:2

8 Gonzalez-Martinez J, Mullin J, Vadera S, Bulacio J, Hughes G, Jones S, et al. Stereotactic placement of depth electrodes in medically intractable epilepsy. J Neurosurg. 2014 Mar; 120(3):639-44.

9 Jung TY, Kim IY, Jung S, Jang WY, Moon KS, Park SJ, et al. Alternative treatment of stereotactic cyst aspiration and radiosurgery for cystic brain metastases. Stereotact Funct Neurosurg. 2014;92(4):234-41.
10 Dammers R, Haitsma IK, Schouten JW, Kros JM, Avezaat CJ, Vincent AJ. Safety and efficacy of frameless and frame-based intracranial biopsy techniques. Acta Neurochir. 2008 Jan;150(1):23-9.

11 Widmann G, Schullian P, Ortler M, Bale R. Frameless stereotactic targeting devices: technical features, targeting errors and clinical results. Int J Med Robot. 2012 Mar;8(1): $1-16$.

12 Ringel F, Ingerl D, Ott S, Meyer B. VarioGuide: a new frameless image-guided stereotactic system - accuracy study and clinical assessment. Neurosurgery. 2009 May;64(5 Suppl 2):365-71.

13 Verploegh IS, Volovici V, Haitsma IK, Schouten JW, Dirven CM, Kros JM, et al. Contemporary frameless intracranial biopsy techniques: might variation in safety and efficacy be expected? Acta Neurochir. 2015 Nov;157(11):2011-6.

14 Bradac O, Steklacova A, Nebrenska K, Vrana J, de Lacy P, Benes V. Accuracy of VarioGuide frameless stereotactic system against framebased stereotaxy: prospective, randomized, single-center study. World Neurosurg. 2017 Aug;104:831-40.

15 Pfisterer WK, Papadopoulos S, Drumm DA, Smith K, Preul MC. Fiducial versus nonfiducial neuronavigation registration assessment and considerations of accuracy. Neurosurgery. 2008 Mar;62(3 Suppl 1):201-7.

16 Sharma M, Deogaonkar M. Accuracy and safety of targeting using intraoperative "O-arm" during placement of deep brain stimulation electrodes without electrophysiological recordings. J Clin Neurosci. 2016 May;27:80-6.
17 Carl B, Bopp M, Saß B, Nimsky C. Intraoperative computed tomography as reliable navigation registration device in 200 cranial procedures. Acta Neurochir. 2018 Sep;160(9): 1681-9.

18 Faust K, Schneider GH, Vajkoczy P. Utilization of the Intraoperative Mobile AIRO ${ }^{\circledR} \mathrm{CT}$ Scanner in Stereotactic Surgery: workflow and Effectiveness. Stereotact Funct Neurosurg. 2019;97(5-6):303-12.

19 Almeida CC, Uzuner A, Alterman RL. Stereotactic Drainage of Brainstem Abscess With the BrainLab Varioguide ${ }^{\mathrm{TM}}$ System and the Airo $^{\mathrm{TM}}$ Intraoperative CT Scanner: Technical Case Report. Oper Neurosurg. 2018 Apr; 14(4):E46-50.

20 Huda W, Magill D, He W. CT effective dose per dose length product using ICRP 103 weighting factors. Med Phys. 2011 Mar;38(3): $1261-5$.

21 Dorward NL, Alberti O, Palmer JD, Kitchen ND, Thomas DG. Accuracy of true frameless stereotaxy: in vivo measurement and laboratory phantom studies. Technical note. J Neurosurg. 1999 Jan;90(1):160-8.

22 Nimsky C, Grummich P, Sorensen A, Fahlbusch R, Ganslandt O. Visualization of the pyramidal tract in glioma surgery by integrating diffusion tensor imaging in functional neuronavigation. Zentralbl Neurochir. 2005; 66(3):133-41.

23 Widmann G, Stoffner R, Sieb M, Bale R. Target registration and target positioning errors in computer-assisted neurosurgery: proposal for a standardized reporting of error assessment. Int J Med Robot. 2009 Dec;5(4):35565. 
24 Nimsky C, Kuhnt D, Ganslandt O, Buchfelder M. Multimodal navigation integrated with imaging. Acta Neurochir Suppl. 2011;109: 207-14.

25 Mascott CR, Sol JC, Bousquet P, Lagarrigue J, Lazorthes Y, Lauwers-Cances V. Quantification of true in vivo (application) accuracy in cranial image-guided surgery: influence of mode of patient registration. Neurosurgery. 2006 Jul;59(suppl 1):ONS146-56.

26 Gempt J, Buchmann N, Ryang YM, Krieg S, Kreutzer J, Meyer B, et al. Frameless imageguided stereotaxy with real-time visual feedback for brain biopsy. Acta Neurochir. 2012 Sep;154(9):1663-7.

27 Buchalla R, Hopf-Jensen S, Rubarth O, Börm W. Frameless navigated biopsy with the BrainLAB ${ }^{\circledR}$ VarioGuide system: a technical note. J Neurol Surg A Cent Eur Neurosurg. 2013 Sep;74(5):321-4.

28 Smith JS, Quiñones-Hinojosa A, Barbaro NM, McDermott MW. Frame-based stereotactic biopsy remains an important diagnostic tool with distinct advantages over frameless stereotactic biopsy. J Neurooncol. 2005 Jun; 73(2):173-9.
29 Zhang Y, Li X, Segars WP, Samei E. Comparison of patient specific dose metrics between chest radiography, tomosynthesis, and CT for adult patients of wide ranging body habitus. Med Phys. 2014 Feb;41(2):023901.

30 Moriarty TM, Quinones-Hinojosa A, Larson PS, Alexander E 3rd, Gleason PL, Schwartz RB, et al. Frameless stereotactic neurosurgery using intraoperative magnetic resonance imaging: stereotactic brain biopsy. Neurosurgery. 2000 Nov; 47(5):1138-45.

31 Neumann JO, Campos B, Younes B, Jakobs M, Jungk C, Beynon C, et al. Frame-based stereotactic biopsies using an intraoperative MR-scanner are as safe and effective as conventional stereotactic procedures. PLoS One. 2018 Oct;13(10):e0205772.

32 Lu Y, Yeung C, Radmanesh A, Wiemann R, Black PM, Golby AJ. Comparative effectiveness of frame-based, frameless, and intraoperative magnetic resonance imaging-guided brain biopsy techniques. World Neurosurg. 2015 Mar;83(3):261-8.
33 Czyż M, Tabakow P, Weiser A, LechowiczGłogowska BE, Zub LW, Jarmundowicz W. The safety and effectiveness of low field intraoperative MRI guidance in frameless stereotactic biopsies of brain tumours-design and interim analysis of a prospective randomized trial. Neurosurg Rev. 2014 Jan; 37(1):127-37.

34 Khatab S, Spliet W, Woerdeman PA. Frameless image-guided stereotactic brain biopsies: emphasis on diagnostic yield. Acta Neurochir. 2014 Aug;156(8):1441-50.

35 Bradáč O, Štekláčová A, Kramáŕ F, Beneš V. Stereotactic brain biopsies using Varioguide system - 101 cases experience. Ces Slov Neurol Neurochir. 2016;79/112(6):579-84.

36 Mader MM, Rotermund R, Martens T, Westphal M, Matschke J, Abboud T. The role of frameless stereotactic biopsy in contemporary neuro-oncology: molecular specifications and diagnostic yield in biopsied glioma patients. J Neurooncol. 2019 Jan;141(1):18394. 